\title{
Navigating the impact of COVID-19 on palliative care for head and neck cancer
}

Pankaj Chaturvedi, MS, FACS ${ }^{1}$

${ }^{1}$ Tata Memorial Center and HBNI

April 28, 2020

\section{Authors:}

Arjun Gurmeet Singh MDS (Oral and Maxillofacial Surgery), MFDS (Glasgow) Department of Head and Neck Oncology

Tata Memorial Center and HBNI Mumbai, India (Email: arjun193@gmail.com)

Jayita Deodhar, MD Department of Palliative Medicine

Tata Memorial Center and HBNI Mumbai, India (Email: jukd2000@yahoo.co.uk)

Pankaj Chaturvedi, MS, FACS, (Corresponding Author) Department of Head and Neck Oncology

Tata Memorial Center, Mumbai, India (Email: chaturvedi.pankaj@gmail.com)

\section{Funding statement}

No funding was obtained from any sources.

\section{Conflict of Interest statement}

None of the authors have any conflict of interest to declare.

\section{Authors contribution statement}

All authors have made a significant contribution to this article from concept to implementation and publication.

\section{Abstract}

Healthcare services are being confronted by a daily dilemma of who can receive critical care and who cannot. In a palliative care clinic, this apprehension gets exemplified, as these patients have limited life expectancy. The head and neck region further makes things critical, as it comprises of all the sites through which the SARS-CoV-2 can be transmitted. This document strives to define the ways in which the head and neck cancer services can contribute to better patient care in a triage context. Practical steps suggested are protective equipment use, ensuring access to critical drugs (such as opioids), greater use of telemedicine consultations, discussing advance care plans and embracing the role of a wider community support.

Keywords: COVID-19, Coronavirus, Palliative care, Head Neck, pandemic

Introduction

Less than a decade ago, the Worldwide Palliative Care Alliance and the World Health Organization (WHO) declared palliative care as a human right.(1) But still in the best times, palliative services are under resourced world over. As health systems are strained by the ongoing pandemic, providing this essential service has 
become an unaddressed ethical challenge. Healthcare services are being confronted by a daily dilemma of who can receive critical care and who cannot. In palliative care services, this apprehension gets exemplified, as these patients have limited life expectancy.

In some sense, both COVID-19 and head and neck cancer patients receiving palliative care run a similar disease course. Resources are frugally rationed, time is always short, patients deteriorate quickly and families are counseled regarding the grim prognosis. What's worse is when a patient receiving palliative care contracts the severe acute respiratory syndrome coronavirus-2 (SARS-CoV-2), it will result in isolation measures extending to the inevitable.

The head and neck region further makes things critical, as it comprises of all the sites through which the SARS-CoV-2 can be transmitted - oral, oropharyngeal, nasal and laryngopharyngeal. Most guidelines suggest that healthcare workers dealing with these sites either defer management of these patients or use appropriate protective measures.(2) This has led to many centers rescheduling appointments indefinitely causing a significant delay in their treatment. This scenario is not limited to low- and middle-income countries with most western countries facing similar if not worse shortages of critical care. In a time where hospital resources are sparse even to treat patients requiring definitive care, how does one provide palliative care? This document strives to define the ways in which the head and neck cancer services can contribute to better patient care in a triage context.

Approaches to respond

The primary goal of all head and neck oncology centers today is to triage patients and avoid unwanted hospitalizations and intensive care that would aggravate the need for rationing services. Most of the centers across the world have implemented some form of contactless appointments, be it virtual or telephonic. Routine home care through these approaches can be very effective in triaging patients early. Whenever possible the referral system should be decentralized to accommodate patients at freestanding hospice or home care centers. To encourage this, certain administrations have relaxed rules whereby hospices can provide appropriate services to insured patients under their medicare and medicaid packages.(3) However, this would lead to hospice services facing an excessive amount of referrals, which could in turn require their own triaging systems and use of teleconsultation. Prescriptions can also be given for longer periods so that patients do not need to return in short intervals. Palliative clinics have also started allocating resources, including personnel, more efficiently in preparation for the pandemic.

Education and Counseling

All measures undertaken should be aimed at preserving and prolonging life. Most people infected with SARS$\mathrm{CoV}-2$ will survive and recover. For those dying as a consequence of the infection who do not wish to have active or invasive treatments, the switch in focus to high quality, compassionate care at the end of their life is equally important. Hence it is of utmost consequence to explore and documented the preferences on further management and goals of care. Patients with decision-making capacity should be appropriately counseled before embarking on any procedure, highlighting the heightened risk of contracting the SARS-CoV-2 virus and possibly disseminating in the community. If the patient/relatives express a wish for hospitalization, they should be informed of the fact that the admission may not be ultimately be put into practice if there is a lack of medical indication, particularly in a situation of scares resources. Few guidelines have suggested a "three talk" approach that includes a team talk, option talk and a decision talk.(4) In addition to educating and counseling patients, palliative care providers should coach the front line staff on methods to deal with negative emotions and communicating empathy. If the disease course can ensue a delay of several days, the physician should decide on medical indication for a local hospital or hospice transfer depending on the following:

- Severity and complexity of the symptoms and additional care needs

- On the nursing home/ambulatory care capacity of delivering quality palliative care

- Availability of palliative care unit resources

- Patient's request 


\section{Emergency department visits}

Pain and dyspnea are the most common complaints that patients receiving palliative care present with to the emergency department.(5) Both COVID-19 and non-COVID-19 conditions (lung metastasis, superior vena cava obstruction, upper airway tumors, etc.) can cause distress or breathlessness towards the end of life. By virtue of its anatomy, head and neck patients can pose a serious threat to the attending healthcare workers. All patients should be treated as potentially COVID-19 positive. Non-invasive approaches should be attempted where possible. Cough hygiene should also minimize the risk of transmission. Standard practice guidelines for intubation and emergency tracheostomy for COVID-19 positive or unknown patient should be followed.(6) Emphasis should be placed on the safety of medical personnel through proper use of personal protective equipment. Once airway is established, suctioning should be done on a closed system and a trach collar is preferred. Patients should not be admitted in the hospital for prolonged periods and dealt with on an outpatient basis.

In general, a symptom-based approach should be adopted. It is imperative to correct what is correctable either through non-drug approaches for mild to moderate disease and drug approaches when required, for example prescribe antibiotics for a bacterial infection. In case of fever, few guidelines have suggested to withhold ibuprofen as concerns have been raised about its possible role in COVID-19.(4) Alternate nonsteroidal anti-inflammatory drugs may be prescribed.

Pain management

Pain management is an integral part of palliative care. Long before the coronavirus hit us, the opioid crisis has been a global controversy. The WHO estimated that $80 \%$ of the world has insufficient access to the required opioid analgesic.(1) With supply chains being affected around the world there have been severe shortages of intensive care drugs, such as morphine, at hospitals treating the COVID-19.(7) This has precipitated in an acute shortage of these critical pain medications among palliative patients. Other opioid substitutes such as buprenorphine and methadone are now being handed out without prescription during the COVID-19 crisis in some countries as a harm reduction strategy for people who are dependent on opioids.(8) This could further exhaust the supply of essential drugs needed for pain relief among these countries. Even though it has been established that opioids are necessary for cancer pain, a large part of palliative care patients will face barriers to relief of such suffering.(9)

During this pandemic, careful triaging of head and neck patients intended to or currently receiving palliative care is necessary to prevent further denuding of critical care resources. Early referrals, where patients are not having a significant symptom burden, could be temporarily avoided during the crisis. This would allow palliative care providers to focus on urgent needs for consults in situations such as management of severe/refractory symptoms, shared decision making and managing anticipatory grief. There is a troubling amount we still do not know about the SARS-CoV-2 infection. One thing certain is that the disease poses a serious hazard to those with compromised immune systems such as cancer patients. In this vulnerable population, COVID-19 could be much worse and more fatal than the cancer itself. The WHO has issued guidance on maintain essential health services during the pandemic with a focus on maternal care, immunization and emergency services among others, but no mention of palliative care.(10) A pandemic is often a powerful amplifier of suffering through physical and mental distress, and financial and social instability. As head and neck cancer care providers it is imperative we deliver the best possible care to patients with the resources at hand, irrespective of their outcomes. In this time of crises, we need to remember to withdraw life sustaining treatments not life sustaining care, and deliver comfort focused treatments and not comfort focused care.

Resources for patients and healthcare providers:

1. Worldwide Hospice and Palliative Care Alliance http://www.thewhpca.org/covid-19

2. International Association of Hospice and Palliative Care (IAHPC) Palliative Care and Covid-19 http://globalpalliativeca 19/

3. COVID-19 Communication Tools from https://docs.google.com/document/d/1uSh0FeYdkGgHsZqem552iC0KmXIgaG 
4. Center to Advance Palliative Care (CAPC) COVID-19 Toolkit https://www.capc.org/toolkits/covid19-response-resources/

5. American Academy of Hospice and Palliative Care COVID-19 Resource http://aahpm.org/education/covid19-resources

References

1. Connor SR, Bermedo MCS, Worldwide Palliative Care Alliance, World Health Organization. Global Atlas of Palliative Care at the End of Life. 2014.

2. Al-Shamsi HO, Alhazzani W, Alhuraiji A, Coomes EA, Chemaly RF, Almuhanna M, et al. A Practical Approach to the Management of Cancer Patients During the Novel Coronavirus Disease 2019 (COVID-19) Pandemic: An International Collaborative Group. The Oncologist. 2020 Apr 3;

3. Medicare Hospice Benefits. :20.

4. C0081-Speciality-guide-Palliative-care-and-coronavirus-FINAL-02.04.20.pdf [Internet]. [cited 2020 Apr 11]. Available from: https://www.england.nhs.uk/coronavirus/wp-content/uploads/sites/52/2020/03/C0081Speciality-guide-Palliative-care-and-coronavirus-FINAL-02.04.20.pdf

5. LAWSON BJ, BURGE FI, MCINTYRE P, FIELD S, MAXWELL D. Palliative Care Patients in the Emergency Department. J Palliat Care. 2008;24(4):247-55.

6. al CPH et. Tracheostomy protocols during COVID-19 outbreak [Internet]. Authorea, Inc.; [cited 2020 Apr 11]. Available from: https://www.authorea.com/users/5588/articles/440747-tracheostomy-protocolsduring-covid-19-outbreak?commit=f29980e67f6730a9d022fae663ae26ff0a8a9851

7. Crucial medicines protected for coronavirus (COVID-19) patients [Internet]. GOV.UK. [cited 2020 Apr 11]. Available from: https://www.gov.uk/government/news/crucial-medicines-protected-for-coronaviruscovid-19-patients

8. correspondent JGH affairs. Methadone to be supplied without new prescription during Covid-19 crisis. The Guardian [Internet]. 2020 Apr 8 [cited 2020 Apr 11]; Available from: https://www.theguardian.com/politics/2020/apr/08 to-be-handed-out-without-prescription-during-covid-19-crisis

9. Cherny NI, Baselga J, de Conno F, Radbruch L. Formulary availability and regulatory barriers to accessibility of opioids for cancer pain in Europe: a report from the ESMO/EAPC Opioid Policy Initiative. Ann Oncol Off J Eur Soc Med Oncol. 2010 Mar;21(3):615-26.

10. Maintaining Essential Health Services and Systems [Internet]. [cited 2020 Apr 11]. Available from: https://www.who.int/emergencies/diseases/novel-coronavirus-2019/technical-guidance/maintaining-essentialhealth-services-and-systems 